\title{
Incoherent Neutron Spin-Echo Spectroscopy as an Option to Study Long-Range Lipid Diffusion
}

\author{
Clare L. Armstrong, ${ }^{1}$ Laura Toppozini, ${ }^{1}$ Hannah Dies, ${ }^{1}$ Antonio Faraone, ${ }^{2,3}$ \\ Michihiro Nagao, ${ }^{2,4}$ and Maikel C. Rheinstädter ${ }^{1,5}$ \\ ${ }^{1}$ Department of Physics and Astronomy, McMaster University, Hamilton, ON, Canada L8S 4M1 \\ ${ }^{2}$ NIST Center for Neutron Research, NIST, Gaithersburg, MD 20899-6100, USA \\ ${ }^{3}$ Department of Materials Science and Engineering, University of Maryland, College Park, MD 20742, USA \\ ${ }^{4}$ Center for Exploration of Energy and Matter, Indiana University, Bloomington, IN 47408-1398, USA \\ ${ }^{5}$ Canadian Neutron Beam Centre, National Research Council of Canada, Chalk River, ON, Canada K0J 1J0
}

Correspondence should be addressed to Clare L. Armstrong; armstc5@mcmaster.ca and Maikel C. Rheinstädter; rheinstadter@mcmaster.ca

Received 4 February 2013; Accepted 25 February 2013

Academic Editors: E. Dague and K. Nishizawa

Copyright (C) 2013 Clare L. Armstrong et al. This is an open access article distributed under the Creative Commons Attribution License, which permits unrestricted use, distribution, and reproduction in any medium, provided the original work is properly cited.

\begin{abstract}
Diffusion is the fundamental mechanism for lipids and other molecules to move in a membrane. It is an important process to consider in modelling the formation of membrane structures, such as rafts. Lipid diffusion is mainly studied by two different techniques: incoherent neutron scattering and fluorescence microscopy. Both techniques access distinctly different length scales. While neutron scattering measures diffusion over about 3 lipid diameters, microscopic techniques access motions of lipids over micrometer distances. The diffusion constants which are determined by these two methods often differ by about an order of magnitude, with the neutrons usually seeing a faster lipid diffusion. Different theories are used to describe lipid diffusion in the two experiments. In order to close the "gap" between these two techniques, we propose to study lipid diffusion at mesoscopic length scales using a neutron spin-echo (NSE) spectrometer. We have conducted an experiment in highly oriented, solid supported lipid bilayers to prove the feasibility of performing incoherent NSE on biological samples. Lateral lipid diffusion was measured in a fluid phase model membrane system at a length scale of $12 \AA$. Using the high-energy resolution of the NSE technique, we find evidence for two dynamic processes.
\end{abstract}

\section{Introduction}

Diffusion not only is the primary mechanism for proteins to move through the lipid matrix, but it also plays an important role in the formation of various macromolecular structures, such as lipid rafts and nanoenvironments for proteins. It is commonly accepted that the Brownian motion of lipid molecules over long length scales (length scales larger than the nearest neighbour distance of the lipid molecule) is characterized by a continuous diffusion process [1-5]. Although this is a well-studied fundamental process, our understanding of molecular diffusion in membranes is still being challenged by new results from experiments and simulations. From computer simulations, subdiffusive and ballistic regimes have been predicted on short time scales and correspondingly small distances $[6,7]$. And ballistic lipid motion in fluid membranes has indeed been recently reported from quasielastic neutron scattering (QENS) using a neutron backscattering spectrometer [8]. Furthermore, it has been reported that lipids move coherently in loosely bound clusters, rather than as independent molecules [9-11]. A "hopping" diffusion of lipids into nearest neighbour sites was observed in single supported bilayers [12], and, recently, it has also been suggested that there is a flow-like component to the motion of the lipid molecules over long length scales [11]. In biology, diffusion often occurs in crowded media, which was found to lead to anomalous diffusion $[13,14]$.

The standard techniques currently used to examine diffusion of different membrane components are neutron scattering techniques and fluorescence spectroscopy. Usually, 
the goal of these techniques is to determine the diffusion time, $\tau_{D}$, of the particles of interest in the membrane and their subsequent diffusion coefficient, $D$. As depicted in Figure 1, the two techniques cover distinctly different length and time scales: fluorescence correlation spectroscopy (FCS) covers motions on micrometer length scales and over microsecond time scales, and neutron beams access dynamics on nanometer lengths and nanosecond times. The diffusion constants determined with the two techniques usually differ by about an order of magnitude, neutrons measuring a faster diffusion as compared to FCS.

It is experimentally challenging to close the gap between the two techniques and follow diffusion of lipid molecules and proteins on mesoscopic length scales. A strong effort is currently being made to enhance the resolution of light microscopes using stimulated emission depletion microscopy [15]. A new generation of neutron backscattering instruments is currently being constructed; however, the accessible length scale, usually determined by the beam size and beam divergence at small scattering angles, is limited to $\approx 20 \AA$. The neutron spin-echo (NSE) technique, with its high energy resolution and the possibility to reach scattering vectors, $Q$, corresponding to tens of $\AA$, is a promising candidate to study long-range diffusion.

Neutrons are scattered by the nuclei. Two factors contribute to differences in the atomic nuclei structure of atoms of the same chemical element: (1) different isotopes and (2) different orientations of the nuclear spin (for elements with nonzero nuclear spin). Therefore, even monatomic samples can contain nuclei with different scattering lengths. Coherent scattering is the scattering which would occur if every nucleus in the system had a scattering length equal to the average scattering length, while the incoherent contribution is caused by isotropic scattering from nuclei with different scattering lengths. The coherent and incoherent scattering contributions give insight into different dynamical processes. Coherent scattering reveals information regarding the correlations between the positions of different atomic nuclei at different times. Thus, it is a method of measuring correlations and interactions in a system. Bragg scattering for instance is the result of coherent scattering. Incoherent scattering only involves correlations between the positions of the same nuclei at different times; therefore, it is a measure of the dynamics of the individual particle. Self-diffusion of lipids and proteins is a dynamical process which will exhibit itself in the incoherent contributions to the scattering [16].

The goal of this experiment was to determine the feasibility of studying lateral lipid diffusion in the plane of the membrane using an NSE spectrometer. To separate in-plane from out-of-plane motions, and to ensure that only lateral lipid diffusion is measured, highly oriented membranes were prepared on silicon wafers and the wafers were carefully aligned in the spectrometer. Because the dynamics of the lipid's selfdiffusion will exhibit itself in the incoherent scattering, an incoherent NSE signal must be measured. Protonated membranes hydrated with heavy water were used to enhance the incoherent scattering. We note that, despite the experimental challenges, incoherent NSE experiments have recently been reported $[17,18]$ in aqueous solutions and polymers. We, for

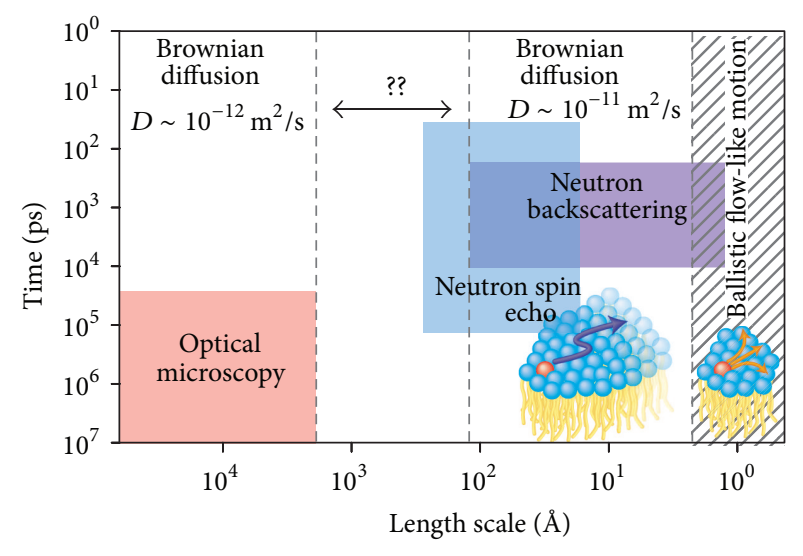

Figure 1: The diffusion of lipids in a bilayer has been measured by various optical and neutron techniques. There still remains a gap (from $\approx 10 \mathrm{~nm}$ to $\approx 200 \mathrm{~nm}$ ) in the characterization of lipid motion between the regimes accessible by fluorescence measurements and neutron backscattering, resulting in a clear difference in the diffusion coefficients observed on each side of this gap. Neutron spin echo (NSE) can access significantly longer length scales than other neutron techniques to give insight into the character of motion at mesoscopic length scales.

the first time, have applied this technique to study lateral diffusion of lipid molecules in a fluid lipid bilayer.

\section{Materials and Methods}

2.1. Sample Preparation. Protonated membranes, hydrated with heavy water, were prepared to maximize the incoherent scattering of the lipid molecules. Highly oriented multilamellar stacks of 1,2-dimyristoyl-sn-glycero-3-phosphocholine (DMPC) were deposited on $2^{\prime \prime}$ (diameter $5.08 \mathrm{~cm}$ ) doubleside polished $\mathrm{Si}$ wafers with a thickness of $300 \mu \mathrm{m}$. A solution of $20 \mathrm{mg} / \mathrm{mL}$ DMPC in 1:1 chloroform and 2,2,2trifluoroethanol (TFE) was prepared. The Si wafers were cleaned by alternate 12 -minute sonications in ultrapure water and methanol at $313 \mathrm{~K}$. This process was repeated twice. As depicted in Figure 2(a), $1 \mathrm{~mL}$ of the lipid solution was pipetted onto each Si wafer and allowed to dry. The wafers were kept in vacuum overnight to remove all traces of the solvent. The samples were then hydrated with heavy water, $\mathrm{D}_{2} \mathrm{O}$, and annealed in an incubator at $308 \mathrm{~K}$ for 24 hours. Following this protocol, each wafer contained roughly 3,000 highly oriented stacked membranes with a total thickness of $\approx 10 \mu \mathrm{m}$.

Eight such Si wafers were stacked with $0.6 \mathrm{~mm}$ aluminium spacers placed in between each wafer to allow the membranes to be hydrated, creating a $10 \%$ scatterer (see Figure 2(b)). The "sandwich" sample was sealed in an aluminium sample can with a $\mathrm{D}_{2} \mathrm{O}$ hydration source.

2.2. Neutron Spin-Echo Experiment. Among neutron scattering spectroscopic techniques, NSE is the one with the highest energy resolution. Figure 3 shows a sketch of a typical NSE experimental setup.

In the most common cases [19], the incoming neutron beam, with a wavelength spread of $\approx 15 \%$, is polarized along 


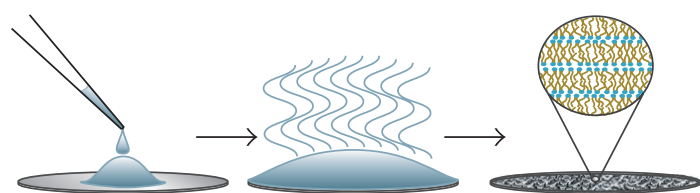

(a)
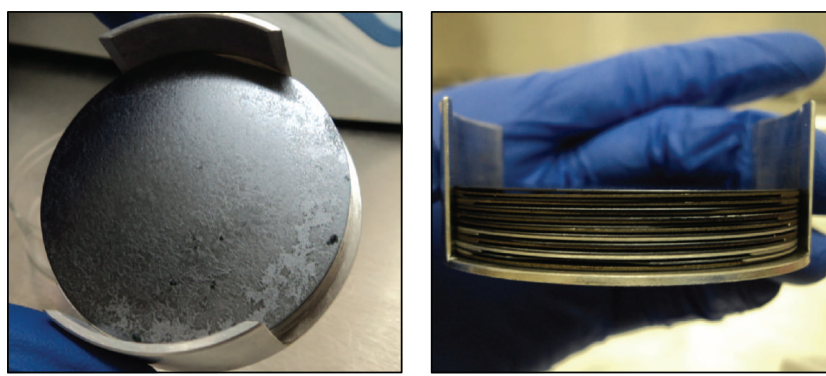

(b)

FIGURE 2: (a) A DMPC solvent solution was deposited onto Si wafers. The solvent was allowed to evaporate completely, resulting in highly oriented bilayers. (b) The wafers were then assembled into a sandwich sample in an aluminium holder with aluminium spacers, which was hydrated with $\mathrm{D}_{2} \mathrm{O}$ from the vapour phase.

the neutrons' velocity direction ( $x$ in Figure 3 ). At the beginning of the instrument, a $\pi / 2$ flipper rotates the spins direction to be perpendicular to the scattering plane (i.e., along $z$ ). Then, the neutron beam travels within the first precession coil through a magnetic field aligned along $x$. Thus, the spins perform a Larmor precession in the $y z$ plane. The angle between the neutron spin direction at the beginning and the end of the first precession coil depends on the time spent within the magnetic field and hence on its velocity. Before being scattered by the sample, the neutron spin is rotated by 180 degrees around the $z$-axis by the $\pi$ flipper. The neutrons are then scattered by the sample and travel through the second precession coil within a field equal to that of the first coil. Inside the second coil, the Larmor precession effectively unwinds the neutron spin. If the scattering event is elastic, the neutron spin direction at the end of the second coil is the same as the one at the beginning of the first coil (which is along the $z$-direction). A second $\pi / 2$ flipper rotates the neutron spin onto the $x y$ plane. The polarization of the beam is finally determined by the analyzerdetector system. Again, if the scattering is elastic, the initial polarization of the scattered beam (which is nominally one) is regained.

When quasielastic scattering occurs the, neutrons' spins do not return to their initial state and it can be shown that the resulting beam polarization is proportional to the sample's intermediate scattering function (ISF), $I(Q, t)$. The value of $t$, often referred to as Fourier time, is proportional to the precession field intensity. Thus, by changing the precession field, NSE can measure the ISF directly in the time domain, whereas other neutron spectrometers, which work in the energy domain, determine the dynamic structure factor, $S(Q, E)$.

Usually, for each investigated Fourier time, the condition of equality between the two precession fields is determined

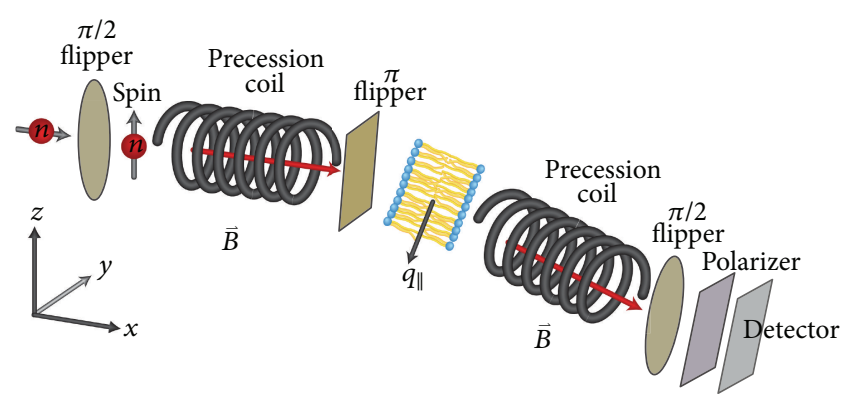

FIGURE 3: Schematic of a NSE experimental setup.

by sweeping the current (phase current) of a coil located inside one of the two spectrometer arms. Thus, the detected intensity follows the typical echo pattern (see Figure 4). The absolute minimum of the echo marks the echo condition for which the two precession fields are the same.

Incidentally, by switching the $\pi / 2$ flippers off the precession, field intensity is essentially set to zero, which implies that the Fourier time value is zero as well. This effectively means that by switching the $\pi / 2$ flippers off and by measuring the $\pi$ flipper ON (spin flip intensity, SF) and OFF (nonspin flip intensity, NSF), a polarized diffraction measurement can be performed without changing the instrumental setup. In this way, the coherent and incoherent scattering intensity can be determined [20].

Because of the requirement of having a homogeneous field along the neutrons' flight path, except for a few noticeable exceptions [21], the NSE detector covers a small fraction of $4 \pi$. Thus, experiments requiring a large $Q$ range coverage are less efficiently performed on an NSE spectrometer, as opposed to a backscattering spectrometer. This is often the case for incoherent scattering experiments where the $Q$ dependence of the scattering function gives precious indications as to the nature of the observed dynamics. Also, the incoherent scattering event has a $2 / 3$ probability of flipping the scattered neutron spin, effectively reducing the incoherent NSE signal by $2 / 3$. However, NSE may have advantages for the study of oriented samples, as will be discussed below.

Using polarized neutrons, it is possible to determine the fraction of coherent and incoherent scattering as a function of the scattering vector, $\vec{Q}$. Unfortunately, in spite of the use of polarized neutrons, NSE cannot separate experimentally the incoherent from coherent dynamics because the neutron spin is employed to encode the energy exchanged with the sample. This limitation is a common issue with other spectrometers which do not use polarized neutrons. With this in mind, as long as the incoherent scattering is dominant, this problem can be overcome by collecting data with statistical significance.

The neutron scattering experiment was performed using the NSE spectrometer, on the Neutron Guide 5 (NG5), at the NIST Center for Neutron Research, Gaithersburg, MD, USA. The NG5-NSE was operated at a wavelength of $\lambda=6 \AA$, with a $\Delta \lambda / \lambda \approx 17.5 \%$.

The sample was placed in a closed cycle refrigerator which can control the sample temperature with an accuracy of 


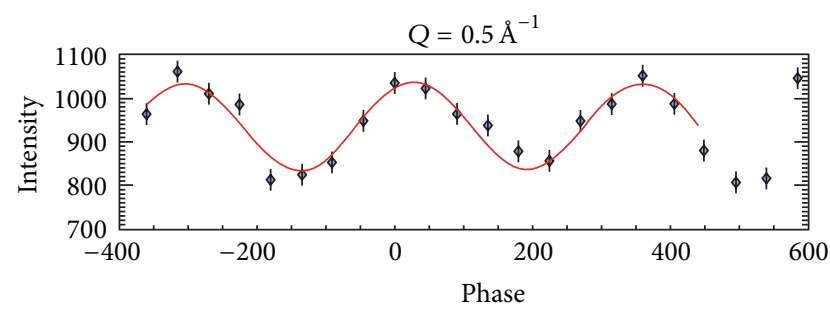

FIgURE 4: An incoherent echo for $q_{\|}=0.5 \AA^{-1}$. The third and second last points report the SF counts (measured twice), whereas the last point represents the NSF counts. The fact that the SF counts are smaller than the NSF counts is a direct evidence that the incoherent scattering is dominant at this $Q$ value. The echo has a negative amplitude which indicates that the observed dynamics come from the incoherent scattering. Error bars shown throughout the paper represent \pm 1 standard deviation.

TABLE 1

\begin{tabular}{lcc}
\hline Peak order & $q_{z}\left(\AA^{-1}\right)$ & $\delta q_{z}$ \\
\hline 1 & 0.1163 & 0.0001 \\
2 & 0.2322 & 0.0009 \\
3 & 0.35 & 0.01 \\
4 & 0.47 & 0.05 \\
\hline
\end{tabular}

$\pm 0.1 \mathrm{~K}$. The sample was mounted vertically in the neutron beam such that the scattering vector $(\vec{Q})$ could either be placed in the membrane plane $\left(q_{\|}\right)$, or perpendicular to the membrane $\left(q_{z}\right)$, by simply rotating the sample by 90 degrees. In this way, both out-of-plane and in-plane structures can easily be measured.

In order to normalize the ISF and to correct the instrumental resolution, $I(Q, t)$ is usually divided by a measurement of a perfectly elastic scatterer. In our case, the resolution was measured by cooling the sample to $70 \mathrm{~K}$ to freeze all molecular motions. The scattering of a sample at such low temperature can be considered to be elastic within the time window of this experiment. The advantage of using the actual sample for normalization is that size and geometry of the resolution sample are preserved. A scan of the empty can was also measured and subtracted from the data. The data were reduced using the software DAVE [22].

2.3. Sample Characterization. The quality of the multilamellar membrane samples was checked prior to the inelastic experiments. The lamellar spacing and mosaicity of the multilamellar membranes were determined by measuring out-ofplane and rocking scans in situ. The NSE spectrometer was used in diffraction mode, with the $\pi / 2$ flippers off, and the data reported are the average of the NSF and SF counts. Figure 5 shows an out-of-plane scan along the $q_{z}$-axis at $T=303 \mathrm{~K}$, in the fluid phase of the membranes. Four pronounced Bragg peaks were observed and their positions were determined from fits of Gaussian peak profiles. The $q_{z^{-}}$ positions are listed in Table 1.

A $d_{z}$-spacing of $54.03 \pm 0.02 \AA$ was determined and this corresponds to a hydration of $99.4 \% \mathrm{RH}$, close to full

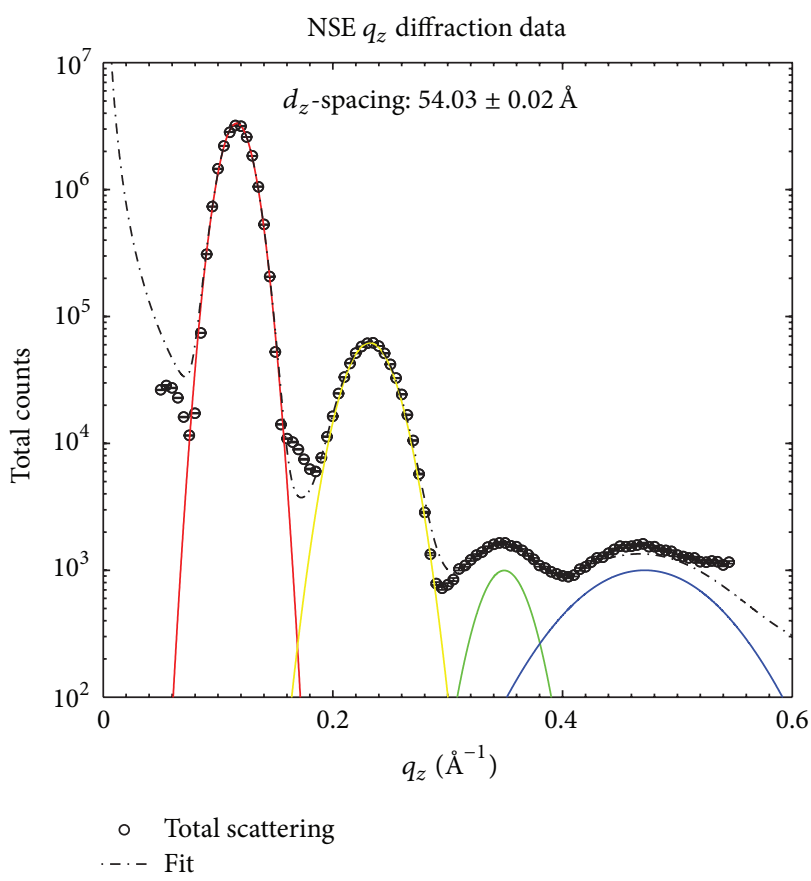

Figure 5: Out-of-plane diffraction data. The $d_{z}$-spacing of $d_{z}=$ $54.03 \AA$, as determined from the fit, corresponds to a hydration of $99.4 \%$ relative humidity $(\mathrm{RH})[23]$

hydration of the bilayers [23]. We note that the out-ofplane Bragg peaks are significantly broadened as compared to measurements on dedicated neutron reflectometers. The reason for this broadening is that NSE spectrometers use a broad wavelength band of $\Delta \lambda / \lambda \approx 17.5 \%$, which leads to an inherent broadening of Bragg reflections. However, the centre of the peaks can well be determined to measure the $d_{z}$-spacing of the stacked membranes.

The quality of the sample was checked in rocking scans, as shown in Figure 6. The distribution of membrane normal vectors can be estimated from the peak width to be smaller than $\approx 1^{\circ}$. The samples are, therefore, characterized as highly oriented membranes. This is a prerequisite to distinguish lateral diffusion from out-of-plane dynamics of the lipid molecules.

\section{Results}

3.1. Polarized Diffraction. The feasibility of detecting incoherent signals critically depends on the ratio between the coherent and incoherent scattering at the different in-plane, $q_{\|}$, positions. Low-angle diffraction was measured and is shown in Figure 7. An empty can, consisting of silicon wafers without membranes, and instrumental background were subtracted from the data. The incoherent scattering of the lipid molecules in the plane of the bilayers is dominant at $q_{\|}$values down to $0.1 \AA^{-1}$, corresponding to a lateral length scale of about $60 \AA$. The packing of the lipid acyl chains in the plane of the membrane leads to a correlation peak at a $q_{\|}$value of $q_{\|} \approx 1.40 \AA^{-1}$ [24-26], with a strong coherent scattering contribution, even in protonated bilayers. This potentially 


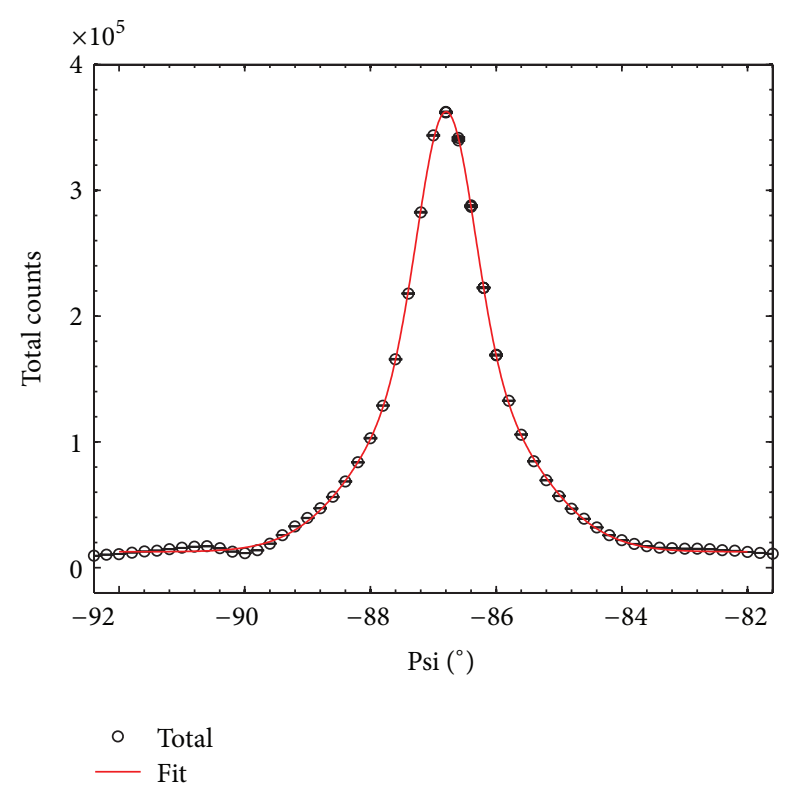

FIGURE 6: Rocking curve centred at $q_{z}=0.117 \AA^{-1}$, the first out-ofplane Bragg peak in Figure 5 The FWHM of the peak was fitted to be $\approx 1^{\circ}$.

leaves a $q_{\|}$range of $0.1 \AA^{-1}<q_{\|}<1.4 \AA^{-1}$, corresponding to length scales of $5 \AA<q_{||}<60 \AA$ for the study of lipid diffusion using incoherent NSE.

For this first test, we have decided to examine dynamics at a $q_{\|}$value of $q_{\|}=0.5 \AA^{-1}$ to prove the feasibility of incoherent NSE experiments in supported membrane systems. Dynamics at this $q_{\|}$value were previously measured using neutron backscattering such that our results could be directly compared in Section 4.

3.2. Incoherent Neutron Spin Echo. A typical spin echo is shown in Figure 4. Because of the weak incoherent signals, long counting times were used. Sample, background, and empty can were measured for $\approx 24$ hours each resulting in a total counting time of $\approx 3$ days at this $q_{\|}$value. Longer counting times can be expected at smaller $q_{\|}$values, where the ratio between incoherent and coherent intensity is less favourable.

The period of the echo is inversely proportional to the wavelength of the neutrons, $\lambda$. By fitting a sinusoidal curve, the amplitude and the average signal are determined. The ISF is obtained from $I\left(q_{\|}, t\right)=(\mathrm{SF}+\mathrm{NSF}) /(\mathrm{SF}-\mathrm{NSF})$. A series of echoes was measured for different spin-echo times, $t$, between 10 picoseconds and 1 nanosecond. The incoherent ISF is shown in Figure 8. Data start at an $I\left(q_{||}, t\right)$ value of $I\left(q_{||}, t\right) \approx$ 0.9 at small times and decays towards longer spin-echo times.

In a first attempt (Figure 8(a)) data were fit using a single exponential decay,

$$
\frac{I\left(q_{\|}, t\right)}{I\left(q_{\|}, 0\right)}=A e^{(-t / \tau)},
$$

in agreement with a single, Brownian diffusion process. However, the corresponding fit in Figure 8(a) does not provide satisfactory agreement with the data.

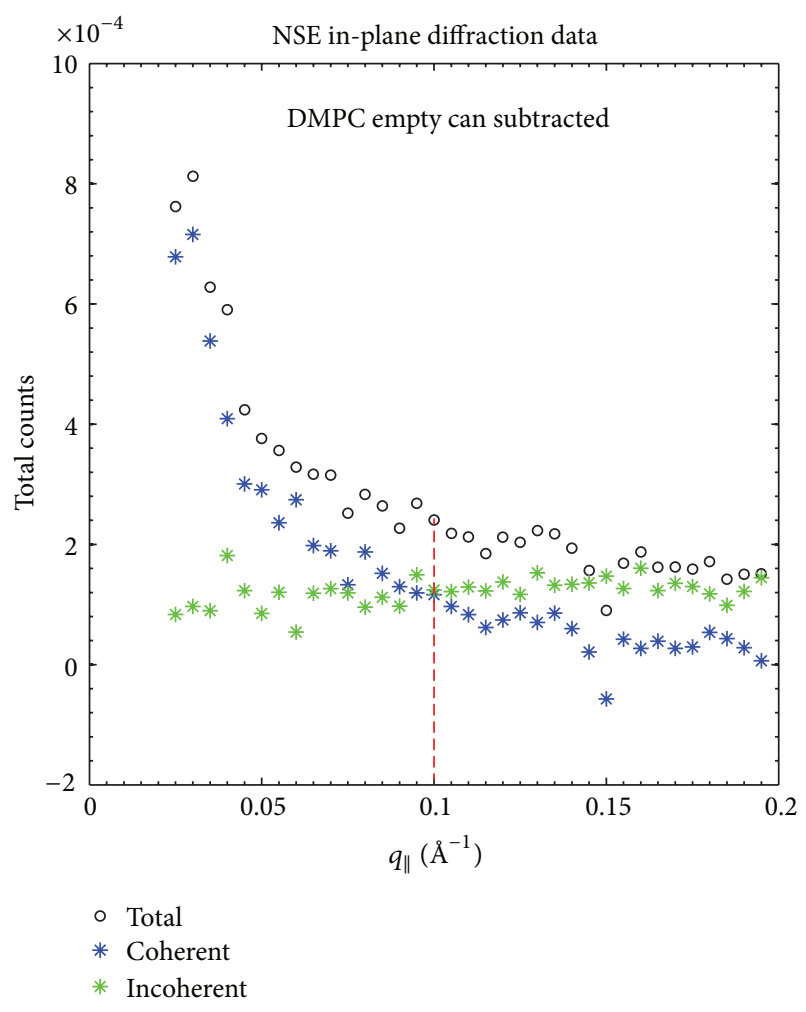

FIGURE 7: Polarized in-plane diffraction data of DMPC with empty can background subtraction. The incoherent scattering signal is dominant above $q_{\|}=0.1 \AA$.

In an attempt to describe the data more adequately, a tentative fit of two exponential functions was used,

$$
\frac{I\left(q_{\|}, t\right)}{I\left(q_{\|}, 0\right)}=A_{1} e^{\left(-t / \tau_{1}\right)}+A_{2} e^{\left(-t / \tau_{2}\right)},
$$

to allow for two dynamical processes. The corresponding fit in Figure 8(b) provides better agreement with the data within the error bars of this experiment. $A_{1}$ and $A_{2}$ are the amplitudes of the two exponential decays; $\tau_{1}$ and $\tau_{2}$ are the corresponding relaxation times. No background was needed to fit the data, which indicates that the incoherent dynamics decay within the time window of this experiment. The fitted parameters are listed in the Table 2.

\section{Discussion}

NSE has been traditionally used to investigate coherent dynamics of polymers and complex systems at $Q$ values corresponding to the small angle region; see [27] for a recent review. However, NSE was recently also successfully used $[17,18]$ to investigate incoherent dynamics. We applied incoherent NSE to study the diffusion of lipid molecules in a fluid lipid membrane. By using long counting times, high quality data could be measured. For this first experiment we have chosen a lateral $q_{\|}$value of $q_{\|}=0.5 \AA^{-1}$ in order to compare the results to those previously obtained by the neutron backscattering technique. 


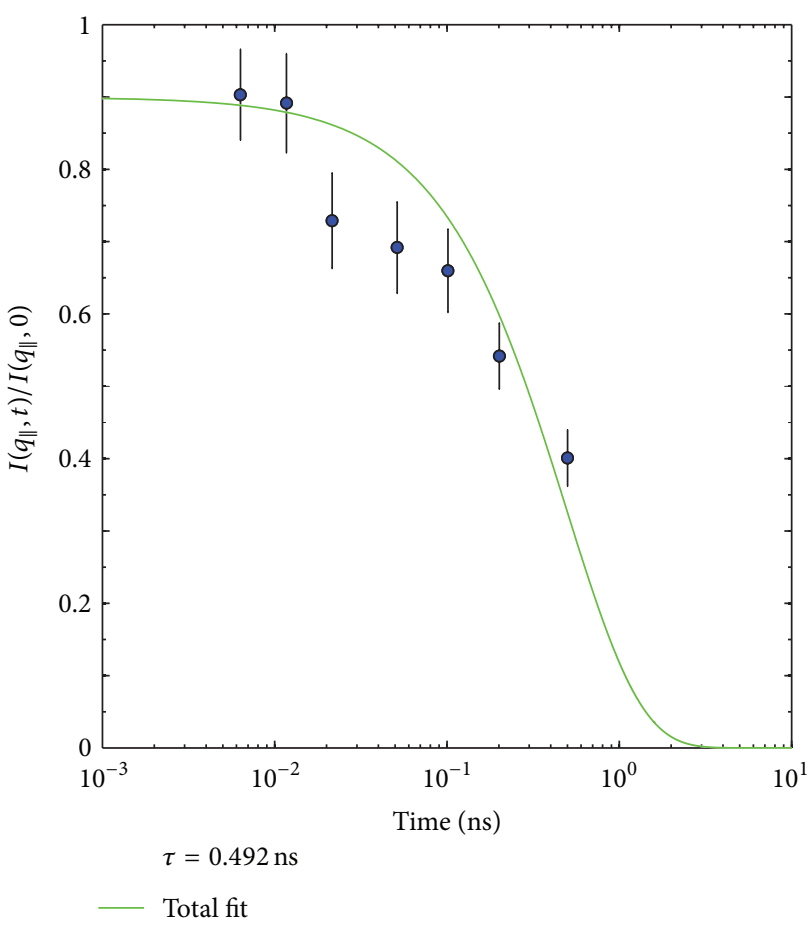

(a)

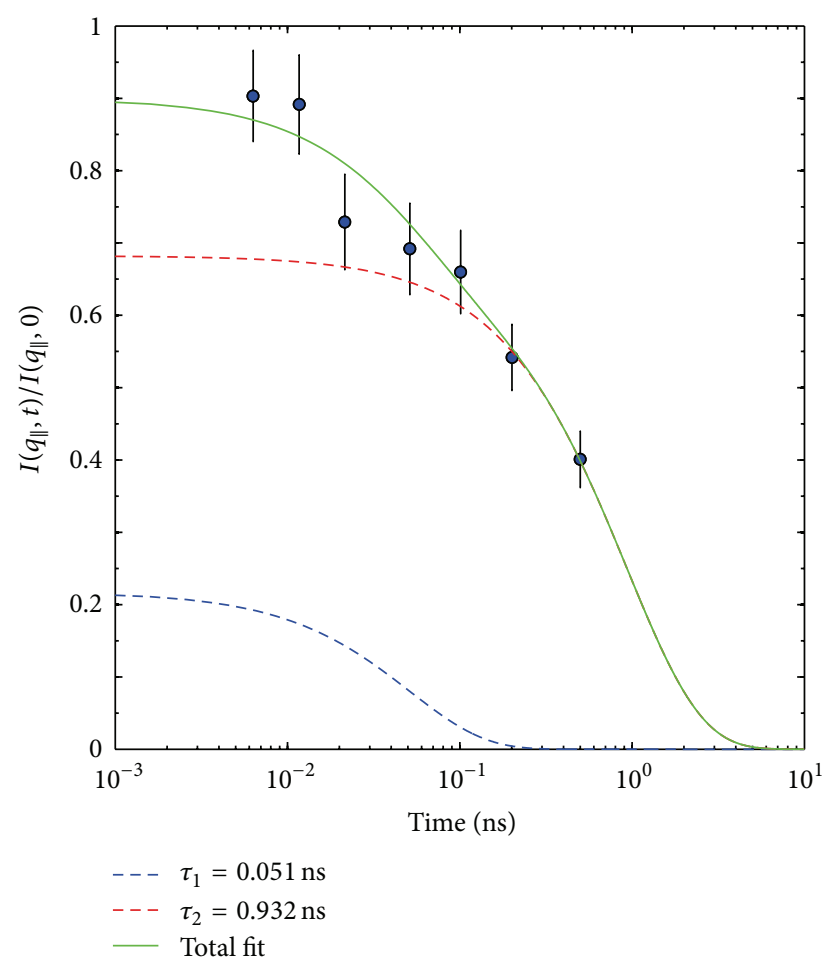

(b)

Figure 8: $I\left(q_{\|}, t\right)$ for $q_{\|}=0.5 \AA^{-1}$. (a) $I\left(q_{\|}, t\right)$ data fit with a single exponential. (b) $I\left(q_{\|}, t\right)$ data fit with a double exponential to individually fit the dynamics occurring at long time scales (dashed red curve) and short time scales (dashed blue curve).

TABLe 2: Parameters used to fit the data shown in Figures 8(a) and 8(b).

\begin{tabular}{|c|c|c|c|c|c|c|c|c|c|c|}
\hline Model & $A_{1}$ & $\begin{array}{c}\tau_{1} \\
\text { (ns) }\end{array}$ & $\begin{array}{l}\delta \tau_{1} \\
\text { (ns) }\end{array}$ & $\begin{array}{c}\Delta E_{1} \\
(\mu \mathrm{eV})\end{array}$ & $\begin{array}{l}\delta \Delta E_{1} \\
(\mu \mathrm{eV})\end{array}$ & $A_{2}$ & $\begin{array}{c}\tau_{2} \\
(\mathrm{~ns})\end{array}$ & $\begin{array}{l}\delta \tau_{2} \\
(\mathrm{~ns})\end{array}$ & $\begin{array}{c}\Delta E_{2} \\
(\mu \mathrm{eV})\end{array}$ & $\begin{array}{l}\delta \Delta E_{2} \\
(\mu \mathrm{eV})\end{array}$ \\
\hline Single exponential & 0.90 & 0.49 & 0.08 & 2.7 & 0.4 & & & & & \\
\hline Double exponential & 0.22 & 0.05 & 0.02 & 26 & 11 & 0.68 & 0.9 & 0.2 & 1.4 & 0.3 \\
\hline
\end{tabular}

Figure 9 shows $\tau_{1}$ and $\tau_{2}$ together with quasielastic energy broadening data previously published for DMPC in its fluid phase, at $T=303 \mathrm{~K}[8]$. Only one diffusion process was observed in the experiment by Armstrong et al. The data quality resulting from this experiment did not allow for the unambiguous assignment of more than one process; however, the diffusion constant obtained from this analysis was in good agreement with coefficients quoted in the literature for similar systems $[1,4,11,12,28]$. The fit in Figure 9 corresponds to a diffusion coefficient of $64 \times 10^{-12} \mathrm{~m}^{2} / \mathrm{s}$. It has been noted that the line of fit in Figure 9 does not pass through the origin, as one would expect from the Brownian diffusion model [8], and the offset is larger than the instrumental resolution. This behaviour is often observed in the literature on experiments using backscattering spectrometers; however, no consistent explanation has been offered.

The relaxation times obtained by NSE were converted to the quasielastic broadening values shown in Table 2 using

$$
\Delta E_{\mathrm{FWHM}}=\frac{2 \hbar}{\tau},
$$

which can be obtained by simply Fourier transforming the self ISF, resulting in a Lorentzian function which has a FWHM that is the quasielastic energy broadening.

This equation can be simplified to

$$
\Delta E_{\mathrm{FWHM}}=\frac{2 \hbar}{\tau} \cdot \frac{10^{15}}{e}=\frac{2}{1.52 \tau},
$$

where the units of $\Delta E_{\mathrm{FWHM}}$ and $\tau$ are $\mu \mathrm{eV}$ and ns, respectively. These energies are included in Figure 9 as stars, which correspond to the two dynamical processes measured at $q_{\|}=$ $0.5 \AA^{-1}$. The relaxation time found by fitting $I\left(q_{\|}, t\right)$ using a single exponential in Figure 8(a) is also included.

The relaxation time determined by the single exponential fit of $\tau=0.49 \mathrm{~ns}$ occurs at a $\Delta E$ value of $\Delta E=2.7 \mu \mathrm{eV}$, much smaller than the process reported by the backscattering technique. However, we note that the corresponding model in Figure 8(a) did not provide a good fit to the data. We, therefore, attempted to fit the data by two exponential functions in Figure 8(b).

The fast process at $\tau_{1}=0.05 \mathrm{~ns}(\Delta E=26 \mu \mathrm{eV})$ agrees well with the dynamics observed in the backscattering experiment 


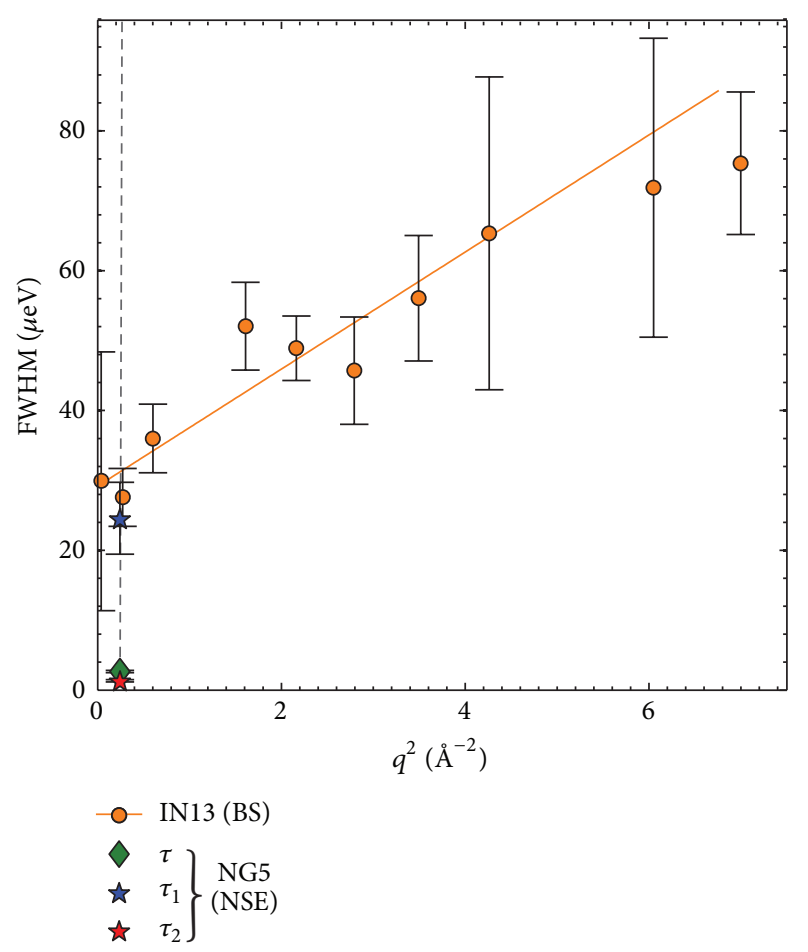

FIGURE 9: Quasielastic energy broadening (FWHM) as function of $q_{\|}^{2}$. The yellow data and fit were obtained from an experiment performed on the same system using neutron backscattering [8]. The diamond corresponds to the energy broadening which relates to the tau value from the single exponential used to fit $I(Q, t)$ in Figure 8(a). The two stars correspond to the tau values obtained from the double exponential fit shown in Figure 8(b).

at this length scale in Figure 9. It, therefore, seems that the energy offset observed in the backscattering experiment is not related to the instrumental resolution of the backscattering instrument, as speculated previously.

An energy offset at small $q_{\|}$has been reported for confined diffusion, such as diffusion in a sphere or cylinder [2931]. At smaller length scales (large $q_{\|}$), the particles follow a typical Brownian diffusion process. However, when the displacement becomes comparable to the size of the confinement, the particles start to "feel" their confinement and the quasielastic energy broadening becomes constant, leading to an offset. The faster process can, therefore, be tentatively assigned to lipid motion confined to a certain area in the membrane. This observation is in agreement with recent results, which suggest that lipids move coherently in loosely bound clusters of $\approx 30 \AA$ diameter, rather than as independent molecules [9-11].

The second, slow process at $\tau_{2}=0.9 \mathrm{~ns}(\Delta E=1.4 \mu \mathrm{eV})$ was not observed in the backscattering data in Figure 9. Slow, long-range diffusion was reported by Busch et al. [11] using the neutron time-of-flight technique. The energy broadening of $\approx 1 \mu \mathrm{eV}$ at a $Q$ value of $0.5 \AA^{-1}$ determined by Bayesian analysis of the small broadening of the instrumental resolution corresponds well with the value for $\tau_{2}$.

The two processes identified in Figure 8(b) can, therefore, be tentatively assigned to a slow, unconstrained lipid diffusion

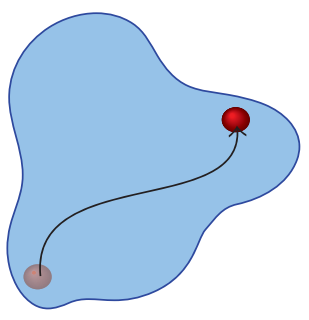

(a)

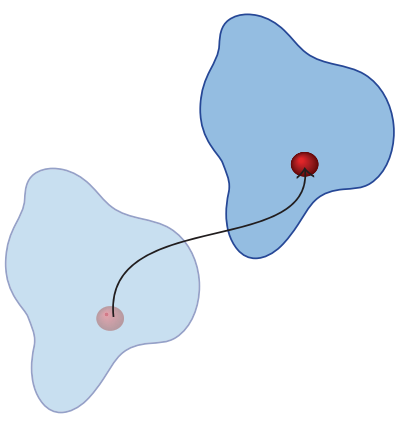

(b)
FIGURE 10: Schematic diagram illustrating the two diffusion processes thought to occur in this system. (a) The faster diffusion process of an individual lipid moving, confined within a domain. (b) The slower diffusion process of a lipid moving as part of a larger domain.

and a second, faster process related to confined lipid diffusion. As depicted in Figure 10, these processes may be related to diffusion of a lipid molecule within a coherently coupled patch, and diffusion of the whole patch. While the confined process (Figure 10(a)) occurs on small length scales, the purely Brownian process in Figure 10(b)) most likely occurs over larger distances and can possibly be observed using FCS.

We note that the purpose of the present experiment was to prove the feasibility of incoherent NSE experiments in solid supported membrane systems and to compare the results to data previously obtained by neutron backscattering. The determination of slopes, from which diffusion coefficients can be calculated, involves the measurement of several $q_{\|}$values, and is beyond the scope of the present experiment. We can not, therefore, make a statement about diffusion constants or the molecular mechanism of diffusion at this time.

Further experiments are required to determine the precise shape of $\Delta E\left(q_{\|}\right)$in an effort to more accurately assign the underlying diffusion mechanisms. To determine the molecular mechanism of lipid diffusion, several $q_{\|}$values between $0.1 \AA^{-1}$ and $1.4 \AA^{-1}$ will be measured in future experiments.

\section{Conclusion}

This experiment proves the feasibility of measuring the diffusion of lipid molecules in oriented membrane systems using incoherent NSE spectrometry.

There are several advantages of using NSE spectrometers to measure diffusion in membranes as compared to backscattering spectrometers. NSE covers a large dynamic range, from $0.005 \mathrm{~ns}$ to $15 \mathrm{~ns}$. By using oriented samples, lateral diffusion can well be separated from out-of-plane motions. And finally, data quality is not limited by the instrumental resolution.

This technique will, in the future, be used to determine lipid diffusion on length scales up to $60 \AA$, a regime previously inaccessible by neutron probes. This length scale is significantly larger than lipid-lipid distances and will allow for the 
testing and designation of different lipid diffusion models to the membrane system.

At present, incoherent NSE experiments in membrane systems are hindered by the inherently long counting times required to resolve the small incoherent signals. However, diffusion of lipids and proteins in membranes under physiological conditions is an important topic in membrane biophysics with implications in cancer and Alzheimer's research, making these types of measurements highly relevant. Incoherent NSE can push the limit of length scales accessible via neutron scattering, thus enabling the study of diffusion over mesoscopic distances and closing the gap between neutron and fluorescence experiments.

\section{Acknowledgments}

This work utilized facilities supported in part by the National Science Foundation under Agreement no. DMR-0944772. This research was funded by the Natural Sciences and Engineering Research Council of Canada (NSERC), the National Research Council Canada (NRC), the Canada Foundation for Innovation (CFI), and the Ontario Ministry of Economic Development and Innovation (OMEDI). H. Dies is the recipient of an NSERC-USRA, and M. C. Rheinstädter is the recipient of an Early Researcher Award of the Province of Ontario.

\section{References}

[1] S. König, W. Pfeiffer, T. Bayerl, D. Richter, and E. Sackmann, "Molecular dynamics of lipid bilayers studied by incoherent quasi-elastic neutron scattering," Journal de Physique II, vol. 2, pp. 1589-1615, 1992.

[2] S. König, E. Sackmann, D. Richter, R. Zorn, C. Carlile, and T. M. Bayerl, "Molecular dynamics of water in oriented DPPC multilayers studied by quasielastic neutron scattering and deuteriumnuclear magnetic resonance relaxation," The Journal of Chemical Physics, vol. 100, no. 4, pp. 3307-3316, 1994.

[3] S. König, T. M. Bayerl, G. Coddens, D. Richter, and E. Sackmann, "Hydration dependence of chain dynamics and local diffusion in L- $\alpha$-dipalmitoylphosphtidylcholine multilayers studied by incoherent quasi-elastic neutron scattering," Biophysical Journal, vol. 68, no. 5, pp. 1871-1880, 1995.

[4] W. Pfeiffer, T. Henkel, E. Sackmann, and W. Knorr, "Local dynamics of lipid bilayers studied by incoherent quasi-elastic neutron scattering," Europhysics Letters, vol. 8, no. 2, p. 201, 1989.

[5] W. Pfeiffer, S. König, J. Legrand, T. Bayerl, D. Richter, and E. Sackmann, "Neutron spin echo study of membrane undulations in lipid multibilayers," Europhysics Letters, vol. 23, no. 6, p. 457, 1993.

[6] E. Flenner, J. Das, M. C. Rheinstädter, and I. Kosztin, "Subdiffusion and lateral diffusion coefficient of lipid atoms and molecules in phospholipid bilayers," Physical Review E, vol. 79, Article ID 011907, 11 pages, 2009.

[7] J. H. Jeon, H. M. S. Monne, M. Javanainen, and R. Metzler, "Anomalous diffusion of phospholipids and cholesterols in a lipid bilayer and its origins," Physical Review Letters, vol. 109, Article ID 188103, 5 pages, 2012.

[8] C. L. Armstrong, M. Trapp, J. Peters, T. Seydel, and M. C. Rheinstädter, "Short range ballistic motion in fluid lipid bilayers studied by quasi-elastic neutron scattering ," Soft Matter, vol. 7, pp. 8358-8362, 2011.

[9] E. Falck, T. Róg, M. Karttunen, and I. Vattulainen, "Lateral diffusion in lipid membranes through collective flows," Journal of the American Chemical Society, vol. 130, no. 1, pp. 44-45, 2008.

[10] M. C. Rheinstädter, J. Das, E. J. Flenner, B. Brüning, T. Seydel, and I. Kosztin, "Motional coherence in fluid phospholipid membranes," Physical Review Letters, vol. 101, Article ID 248106 , 4 pages, 2008.

[11] S. Busch, C. Smuda, L. C. Pardo, and T. Unruh, "Molecular mechanism of long-range diffusion in phospholipid membranes studied by quasielastic neutron scattering," Journal of the American Chemical Society, vol. 132, no. 10, pp. 3232-3233, 2010.

[12] C. L. Armstrong, M. D. Kaye, M. Zamponi et al., "Diffusion in single supported lipid bilayers studied by quasi-elastic neutron scattering," Soft Matter, vol. 6, no. 23, pp. 5864-5867, 2010.

[13] F. Roosen-Runge, M. Hennig, F. Zhang et al., "Protein selfdiffusion in crowded solutions," Proceedings of the National Academy of Sciences of the United States of America, vol. 108, no. 29, pp. 11815-11820, 2011.

[14] D. S. Banks and C. Fradin, "Anomalous diffusion of proteins due to molecular crowding," Biophysical Journal, vol. 89, no. 5, pp. 2960-2971, 2005.

[15] S. W. Hell and J. Wichmann, "Breaking the diffraction resolution limit by stimulated emission: stimulated-emissiondepletion fluorescence microscopy," Optics Letters, vol. 19, no. 11, pp. 780-782, 1994.

[16] M. Bée, Quasielastic Neutron Scattering: Principles and Applications in Solid State Chemistry, Biology and Materials Science, Taylor \& Francis, Boca Raton, Fla, USA, 1988.

[17] E. Mamontov, A. Faraone, E. W. Hagaman, K. S. Han, and E. Fratini, "A low-temperature crossover in water dynamics in an aqueous $\mathrm{LiCl}$ solution: diffusion probed by neutron spin-echo and nuclear magnetic resonance," Journal of Physical Chemistry $B$, vol. 114, no. 50, pp. 16737-16743, 2010.

[18] A. Wischnewski, M. Monkenbusch, L. Willner, D. Richter, and G. Kali, "Direct observation of the transition from free to constrained single-segment motion in entangled polymer melts," Physical Review Letters, vol. 90, no. 5, Article ID 058302, 4 pages, 2003.

[19] F. Mezei, Ed., Neutron Spin Echo, Springer, Berlin, Germany, 1980.

[20] A. M. Gaspar, S. Busch, M. S. Appavou et al., "Using polarization analysis to separate the coherent and incoherent scattering from protein samples," Biochimica et Biophysica Acta, vol. 1804, no. 1, pp. 76-82, 2010.

[21] B. Farago, "IN11C, medium-resolution multidetector extension of the IN11 NSE spectrometer at the ILL," Physica B, vol. 241243, pp. 113-116, 1997.

[22] R. T. Azuah, L. R. Kneller, Y. Qiu et al., "DAVE: a comprehensive software suite for the reduction, visualization, and analysis of low energy neutron spectroscopic data," Journal of Research of the National Institute of Standards and Technology, vol. 114, no. 6, pp. 341-358, 2009.

[23] N. Chu, N. Kučerka, Y. Liu, S. Tristram-Nagle, and J. F. Nagle, "Anomalous swelling of lipid bilayer stacks is caused by softening of the bending modulus," Physical Review E, vol. 71, Article ID 041904, 8 pages, 2005.

[24] M. C. Rheinstädter, C. Ollinger, G. Fragneto, F. Demmel, and T. Salditt, "Collective dynamics of lipid membranes studied by inelastic neutron scattering," Physical Review Letters, vol. 93, no. 10, Article ID 108107, 4 pages, 2004. 
[25] C. L. Armstrong, M. A. Barrett, A. Hiess et al., "Effect of cholesterol on the lateral nanoscale dynamics of fluid membranes," European Biophysics Journal, vol. 41, no. 10, pp. 901-913, 2012.

[26] C. L. Armstrong, M. A. Barrett, L. Toppozini et al., "Coexistence of gel and fluid lipid domains in single-component phospholipid membranes," Soft Matter, vol. 8, pp. 4687-4694, 2012.

[27] D. Richter, M. Monkenbusch, A. Arbe, and J. Colmenero, Neutron Spin Echo in Polymer Systems, vol. 174 of Advances in Polymer Science, Springer, Berlin, Germany, 2005.

[28] A. Buchsteiner, T. Hauß, S. Dante, and N. Dencher, "Alzheimer's disease amyloid- $\beta$ peptide analogue alters the ps-dynamics of phospholipid membranes," Biochimica et Biophysica Acta, vol. 1798, no. 10, pp. 1969-1976, 2010.

[29] F. Volino and A. Dianoux, "Neutron incoherent scattering law for diffusion in a potential of spherical symmetry: general formalism and application to diffusion inside a sphere," Molecular Physics, vol. 41, no. 2, pp. 271-279, 1980.

[30] M. Bée, "Localized and long-range diffusion in condensed matter: state of the art of QENS studies and future prospects," Chemical Physics, vol. 292, no. 2-3, pp. 121-141, 2003.

[31] D. Bicout, "Incoherent neutron scattering functions for diffusion inside two concentric spheres," Physical Review E, vol. 62, no. 1, pp. 261-271, 2000. 

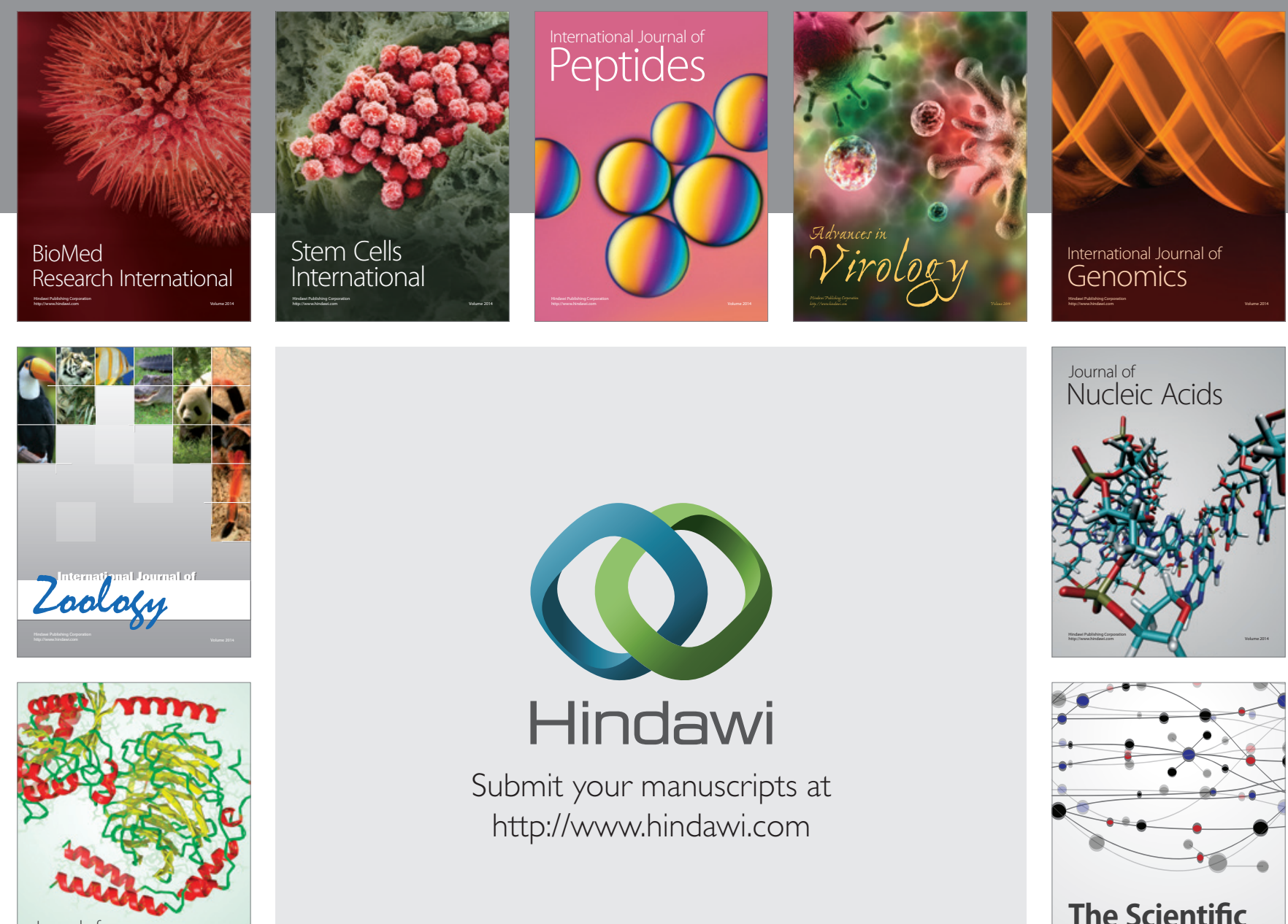

Submit your manuscripts at

http://www.hindawi.com

Journal of
Signal Transduction
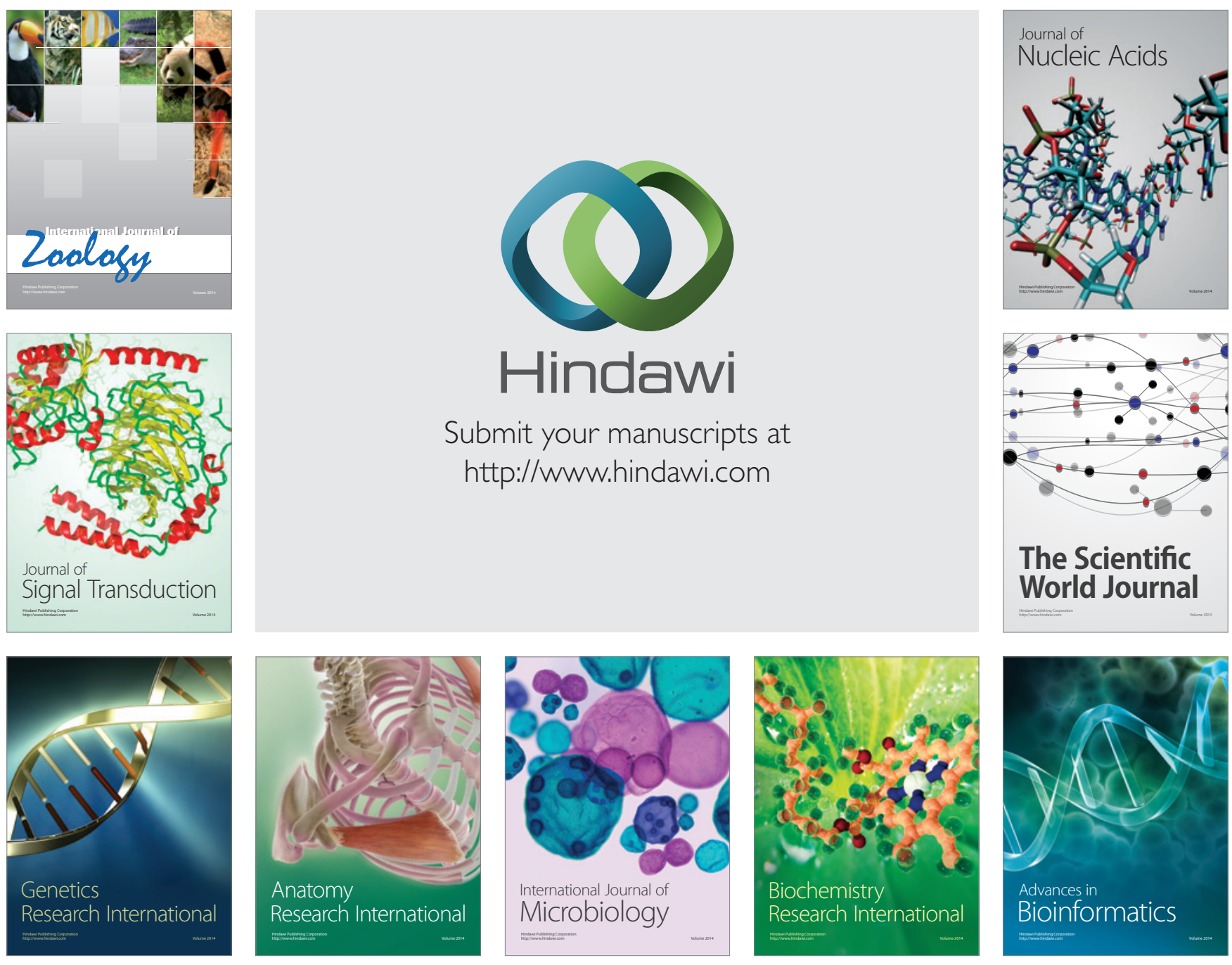

The Scientific World Journal
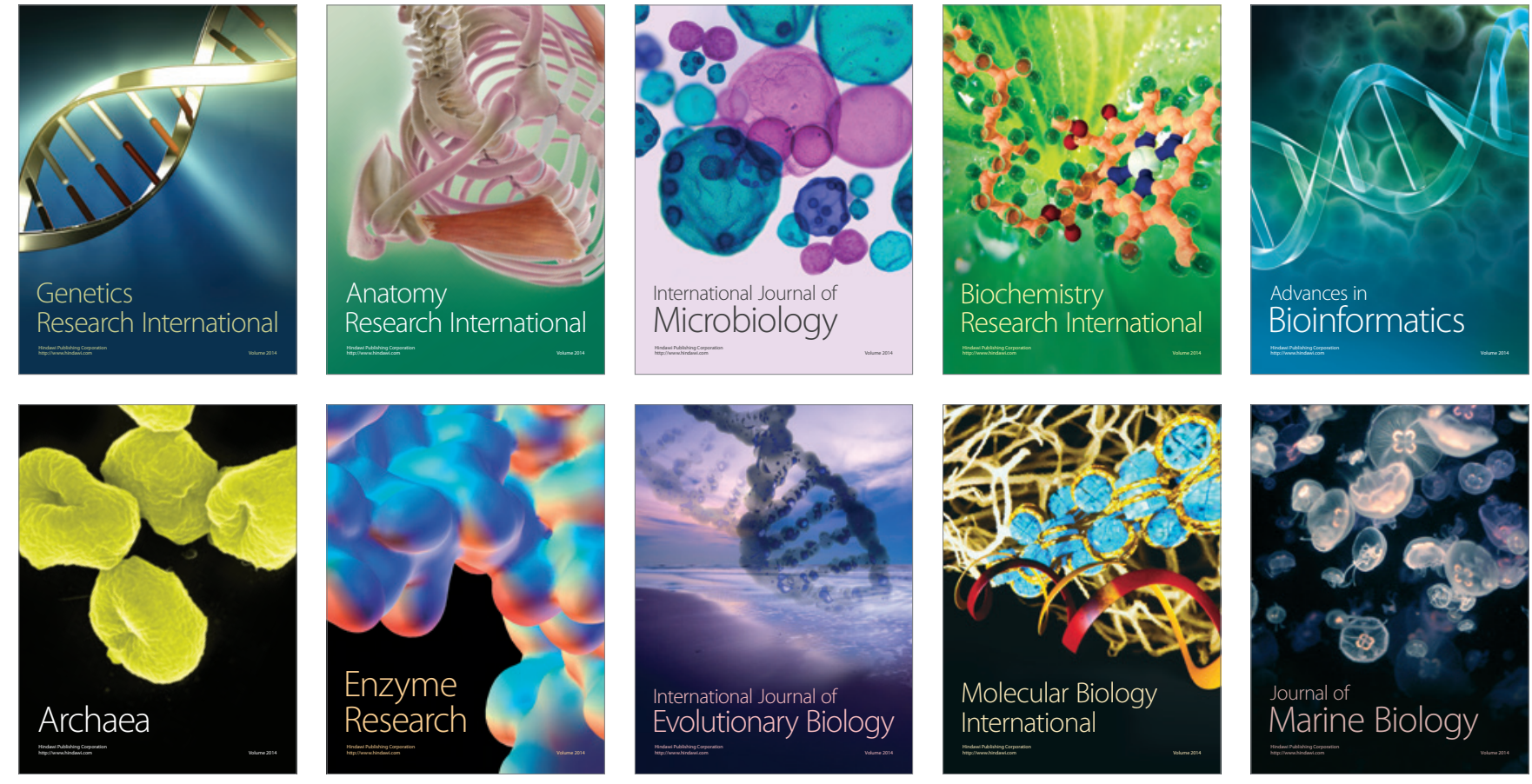